\title{
Expression of NUAK2 in gastric cancer tissue and its effects on the proliferation of gastric cancer cells
}

\author{
LIN TANG $^{1 *}$, SHU-JUAN TONG ${ }^{*}$, ZHEN ZHAN $^{1}$, QIAN WANG ${ }^{1}$, YUAN TIAN $^{2}$ and FENG CHEN ${ }^{2}$ \\ ${ }^{1}$ School of Medicine and Life Sciences, Nanjing University of Chinese Medicine, Nanjing, Jiangsu 210023; \\ ${ }^{2}$ Division of General Surgery, Shanghai Jiao Tong University Affiliated Sixth People's Hospital, Shanghai 200233, P.R. China
}

Received June 19, 2015; Accepted August 16, 2016

DOI: $10.3892 /$ etm.2016.3983

\begin{abstract}
The present study aimed to analyze the expression and effects of NUAK2 in gastric cancer and adjacent normal gastric tissues. The protein expression levels of NUAK2 were detected by western blot analysis. The effects of NUAK2 expression on the proliferation of gastric cancer cells was detected using an MTT and BrdU incorporation assay. Furthermore, the effects of NUAK2 on proliferation and cancer stem cell markers, both protein and microRNA (miRNA), were investigated by western blot analysis and miRNA microarrays, respectively. The results demonstrated that NUAK2 was able to significantly promote the proliferation of SGC-7901 gastric cancer cells. In addition, NUAK2 overexpression decreased the percentage of cells in the G1 phase and increased the percentage of cells in the $\mathrm{S}$ phase. Western blot analysis and miRNA microarrays revealed that overexpression of NUAK2 resulted in increased expression levels of proliferation markers, including c-myc, proliferating cell nuclear antigen, cyclin-dependent kinase 2, miRNA 21, and gastric cancer stem cell markers, including aldehyde dehydrogenase 1 , CD44 and CD133. In conclusion, NUAK2 expression differed between the tumor and normal gastric tissues. NUAK2 was able to promote the proliferation of gastric cancer cells and regulate their cell cycle. Proliferation and cancer stem cell markers were upregulated by NUAK2 expression. Therefore, the results from the present study suggest that NUAK2 may be a promising target for gastric cancer therapy in the future.
\end{abstract}

\section{Introduction}

Gastric cancer is a common malignant tumor with a high mortality rate in Asian countries (1). The molecular patho-

Correspondence to: Dr Feng Chen, Division of General Surgery, Shanghai Jiao Tong University Affiliated Sixth People's Hospital, 222 Huanhuxisan Road, Shanghai 200233, P.R. China

E-mail: chenfengmail@163.com

*Contributed equally

Key words: gastric cancer, NUAK2, microRNA, proliferation genesis of gastric cancer is complex and remains poorly understood; however, several factors are reported to be important in promoting gastric cancer $(2,3)$, including oncogene protein expression (4). These proteins have varying expression levels in tumor and normal gastric tissues, and are able to cause a variety of changes in the biological behavior of cells (5).

NUAK2 is an important gene that regulates cell cycle progression and cell migration in melanoma cells (6). The importance of NUAK2 in melanomagenesis is highlighted by its impact on the survival of patients with acral melanomas (7). In addition, NUAK 2 participates in the regulation of cell proliferation of melanomas (8). A previous study demonstrated that NUAK2 and the phosphoinositide 3-kinase (PI3K) signaling pathway control the expression of cyclin-dependent kinase 2 (CDK2), increase the S-phase population in the cell cycle profile and increase the proliferation of both C32 and SM2-1 melanoma cells (9). However, the expression and function of NUAK2 in gastric cancer has yet to be elucidated.

In the present study, the expression of NUAK2 in gastric cancer was investigated. In addition, an SGC-7901 cell model overexpressing NUAK2 was used to investigate the possible mechanisms underlying the effects of NUAK2 on gastric cancer.

\section{Materials and methods}

Tumor specimens and cell culture. Gastric cancer tissue specimens were collected from eight patients (age, 50-60 years; 3 female, 5 male) at the Shanghai Jiao Tong University Affiliated Sixth People's Hospital (Shanghai, China) from January 2011 to December 2014. Matched non-tumor adjacent tissue was obtained from a segment of the resected specimens that was the farthest from the tumor $(>5 \mathrm{~cm})$. Diagnosis of gastric cancer were based on histopathological evaluation. Written informed consent was obtained from the patients and the study was approved by the Medical Ethics Committees of the Shanghai Jiao Tong University Affiliated Sixth People's Hospital. The SGC-7901 gastric cancer cell line (MD Anderson Cancer Center, Houston, TX, USA) was cultured and maintained as previously described (10).

Western blot analysis. Protein extracts from the cells or tissue samples were prepared by mechanical lysis and homogenization in radioimmunoprecipitation assay buffer 
(Beyotime Institute of Biotechnology, Haimen, China). Protein concentration was detected using a bicinchoninic acid protein assay kit (Beyotime Institute of Biotechnology) The cell lysates (30 $\mu \mathrm{g}$ protein/lane) were separated by $10 \%$ SDS-PAGE and transferred onto polyvinylidene fluoride membranes (GE Healthcare Life Sciences, Chalfont, UK). After blocking 5\% non-fat dry milk in TBS, the membranes were then incubated with the following primary antibodies overnight at $4^{\circ} \mathrm{C}$ : Anti-NUAK2 (cat. no. ab107287), anti-c-myc (cat. no. ab32072), anti-p21 (cat. no. ab47452), anti-p53 (cat. no. ab131442), anti-proliferating cell nuclear antigen (PCNA; cat. no. ab18197), anti-CDK2 (cat. no. ab70012), anti-aldehyde dehydrogenase 1 (ALDH1; cat. no. ab190298), anti-CD44 (cat. no. ab157107), anti- $\beta$-actin (cat. no. ab8226) (all purchased from Abcam, Cambridge, MA, USA and used at a 1:500 dilution) and anti-CD133 (11) (supplied by Tongji Medical College Huazhong University of Science and Technology, Shanghai, China). Incubation with horseradish peroxidase-conjugated anti-mouse (cat. no. sc-2375) and anti-rabbit (cat. no. sc-2385) secondary antibodies (Santa Cruz Biotechnology, Inc., Dallas, TX, USA) was performed for $40 \mathrm{~min}$ at room temperature and enhanced chemiluminescence visualizations were performed as previously described (12).

Vectors and transfection. NUAK2-expressing plasmids and empty vector (pcDNA3.1; provided by Tongji Medical College Huazhong University of Science and Technology) were used. For transfection experiments, the cells were cultured in serum-free medium without antibiotics at $60 \%$ confluence for $24 \mathrm{~h}$, and then transfected with transfection reagent (Lipofectamine 2000; Invitrogen, Carlsbad, CA, USA) according to manufacturer's instructions. After incubation for $6 \mathrm{~h}$, the medium was removed and replaced with normal culture medium for $48 \mathrm{~h}$ (13).

Cell proliferation and cell-cycle assays. The viability of SGC-7901 cells was assessed using an MTT assay (Sigma-Aldrich, St. Louis, MO, USA). SGC-7901 cells were transfected with NUAK2 or pcDNA3.1 (mock). These cells were incubated in a humidified atmosphere with $5 \% \mathrm{CO}_{2}$. Then, MTT solution $(4.14 \mathrm{mg} / \mathrm{ml})$ was added to each well and the optical density was measured after incubation at $37^{\circ} \mathrm{C}$ for $4 \mathrm{~h}$ (14). Absorbance was directly proportional to the number of surviving cells. For the BrdU incorporation assays, a cell proliferation ELISA (Beyotime Institutes of Biotechnology, Inc., Shanghai, China) was used to analyze the incorporation of BrdU during DNA synthesis, following the manufacturer's instructions. Cell cycle profile analyses were performed as previously described (12). Cells $\left(8.0 \times 10^{5}\right)$ were seeded into a $100-\mathrm{mm}$ culture plate and allowed to attach overnight. The cells were transfected with plasmids for $24 \mathrm{~h}$, washed twice with $\mathrm{NaCl} / \mathrm{Pi}$, and then centrifuged at $200 \times g$ at room temperature. The pellet was resuspended in $1 \mathrm{ml}$ cold $\mathrm{NaCl} / \mathrm{Pi}$ and fxed in $70 \%$ ethanol for at least $12 \mathrm{~h}$ at $4^{\circ} \mathrm{C}$. The fixed cells were incubated with $100 \mu$ l DNase-free RNaseA $(200 \mu \mathrm{g} / \mathrm{ml})$ for $30 \mathrm{~min}$ at $37^{\circ} \mathrm{C}$, and then $1 \mathrm{mg} / \mathrm{ml}$ propidium iodide was added. The stained cells were analyzed using a fluorescence-activated cell sorter (BD Accuri C6; BD Biosciences, Ann Arbor, MI, USA). The percentages of cells in the G1, S and G2/M phases of the cell cycle were determined using CellQuest Pro software (FlowJo, Ashland, OR, USA).

RNA extraction and microRNA (miRNA) microarrays. Total RNA was extracted from the cells using TRIzol ${ }^{\circledR}$ reagent (Invitrogen; Thermo Fisher Scientific, Inc.), in accordance with the manufacturer's instructions. miRNA microarrays were performed as previously described $(12,15)$.

Statistical analysis. Data are presented as the mean \pm standard deviation from at least three independent experiments. Differences between groups were analyzed using a Student's $t$-test. Statistical analyses were performed using SPSS 13.0 software (SPSS, Inc., Chicago, IL, USA). P<0.05 was considered to indicate a statistically significant difference.

\section{Results}

NUAK2 is upregulated in gastric cancer tissues. To assess NUAK2 expression levels in gastric cancer, western blot analysis was conducted in eight pairs of gastric cancer tissue samples and matched adjacent normal tissue sample samples. The expression of NUAK2 was higher in the gastric cancer tissue samples compared with the normal tissue samples (Fig. 1). These data indicate that NUAK2, which has an impact on the survival of patients with acral melanomas, may also act as an oncogene in gastric cancer.

NUAK2 overexpression promotes gastric cancer cell proliferation in vitro. Since NUAK2 was observed to be upregulated in gastric cancer tissues, the effect of NUAK2 on gastric cancer proliferation was investigated. SGC-7901 cells were transfected with NUAK2-expressing plasmids or empty vector (Fig. 2A). The results demonstrated that cell proliferation was significantly increased in NUAK2-overexpressing cells compared with that of their corresponding controls, as determined by MTT and BrdU assays (Fig. 2B and C). Furthermore, NUAK2 overexpression decreased the percentage of cells in the G1 phase and increased the percentage of cells in the $\mathrm{S}$ phase (Fig. 2D).

NUAK2 upregulates proliferation and cancer stem cell-associated protein expression. To further explore the function of NUAK2 on tumor proliferation, western blotting was performed to identify whether proliferation markers were also affected by NUAK2 in the cells. The results demonstrated that c-myc, PCNA and CDK2 expression were upregulated, whereas p21 and p53 expression were downregulated by NUAK2 (Fig. 3). In addition, western blotting was also performed to further confirm that NUAK2 was able to regulate cancer stem cell markers. The results demonstrated that the expression of gastric cancer stem cell markers ALDH1 (16), CD44 (17) and CD133 $(18,19)$ were upregulated in cells transfected with NUAK2-expressing plasmids compared with their corresponding controls (Fig. 3). These data suggest that NUAK2 is able to promote the expression of proliferation and cancer stem cell markers in SGC-7901 cells.

NUAK2 upregulates proliferation-associated miRNA expression. Oncogenes or tumor suppressor genes may have 


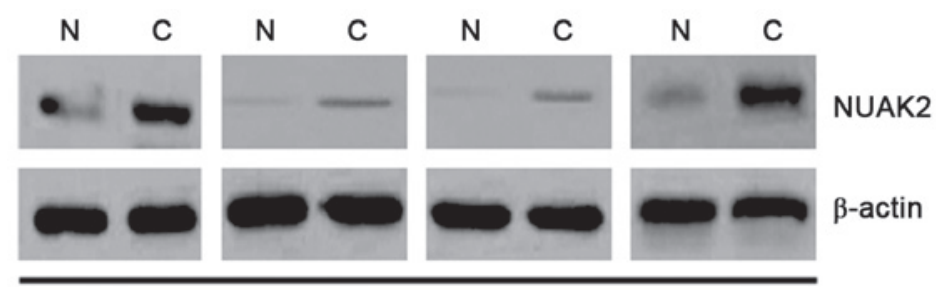

Tissues of gastric cancer patients

(No.1-4)

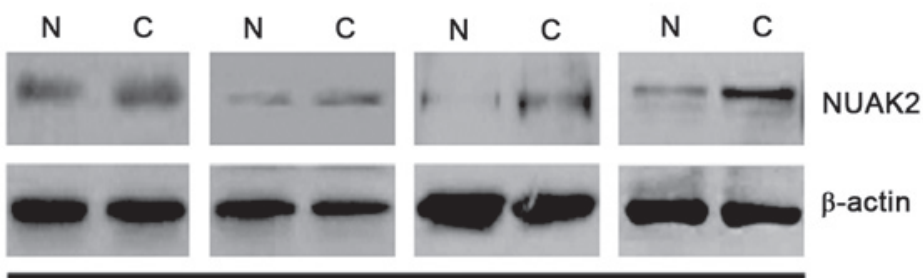

Tissues of gastric cancer patients

(No.5-8)

Figure 1. Protein expression levels of NUAK2 in gastric cancer tissue samples. NUAK2 expression was determined by western blot analysis in eight pairs of human gastric cancer $(\mathrm{C})$ and adjacent normal tissue samples $(\mathrm{N})$.

A

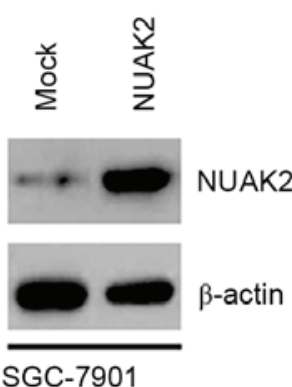

D
B

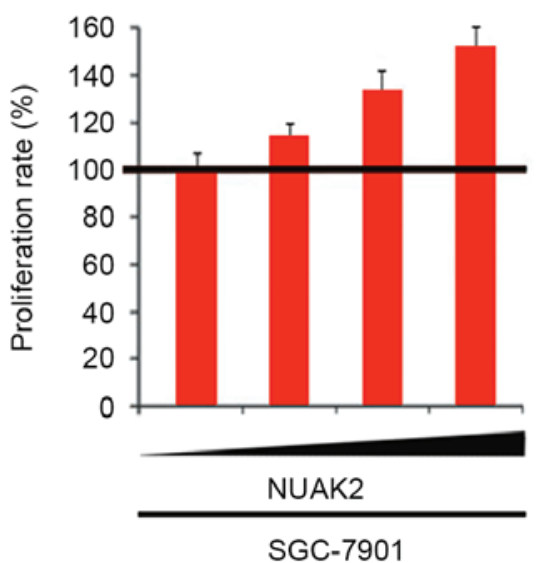

C

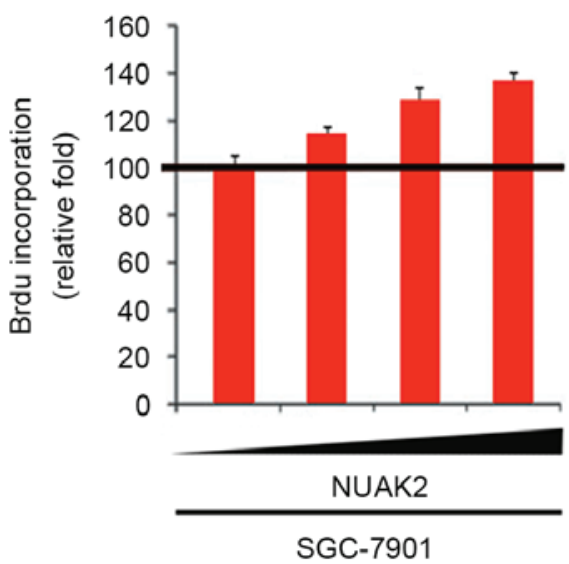

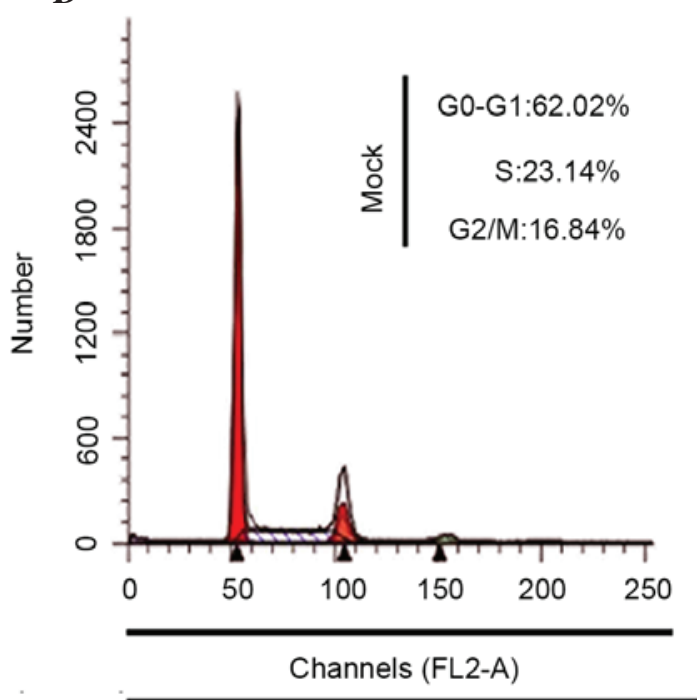

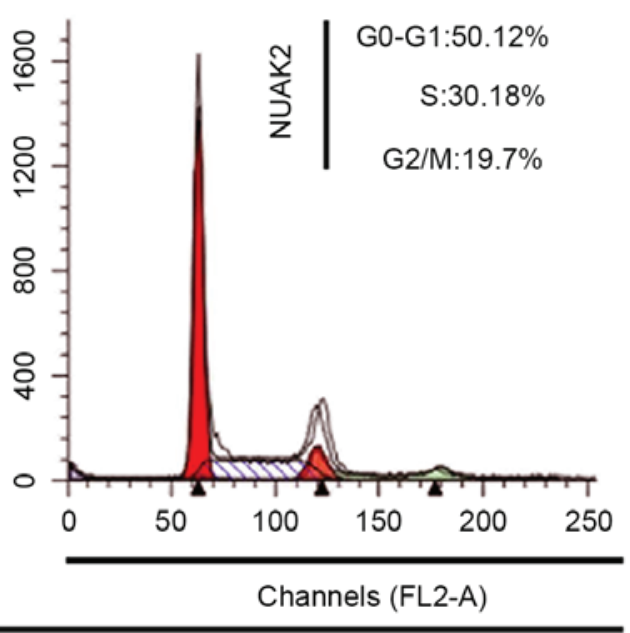

SGC-7901

Figure 2. NUAK2 overexpression promotes proliferation of SGC-7901 gastric cancer cell. (A) Expression levels of NUAK2 in SGC-7901 cells transfected with NUAK2-expressing plasmids or empty vector (mock). (B) The cell viability of SGC-7901 cells following transfection with NUAK2-expressing plasmids or empty vector (mock), analyzed by MTT assay. (C) The proliferative potential of SGC-7901 cells following transfection with NUAK2-expressing plasmids or empty vector (mock), analyzed using Brdu incorporation assay. (D) The cell cycle phases of SGC-7901 cells transfected with NUAK2-expressing plasmids or empty vector (mock) were analyzed by flow cytometry. 


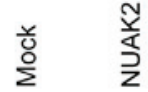
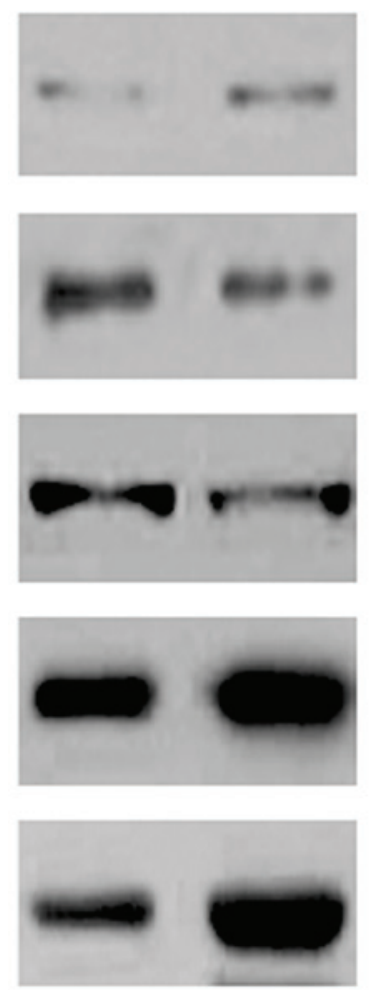

CDK2

p53

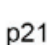

PCNA

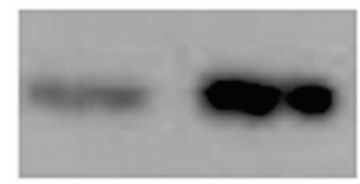

ALDH1

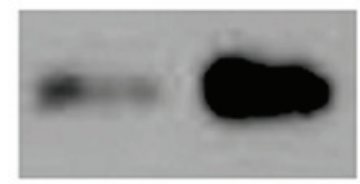

CD44

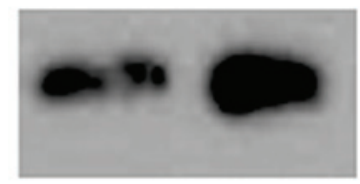

CD133

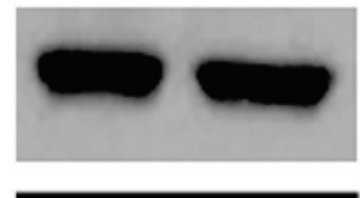

SGC-7901

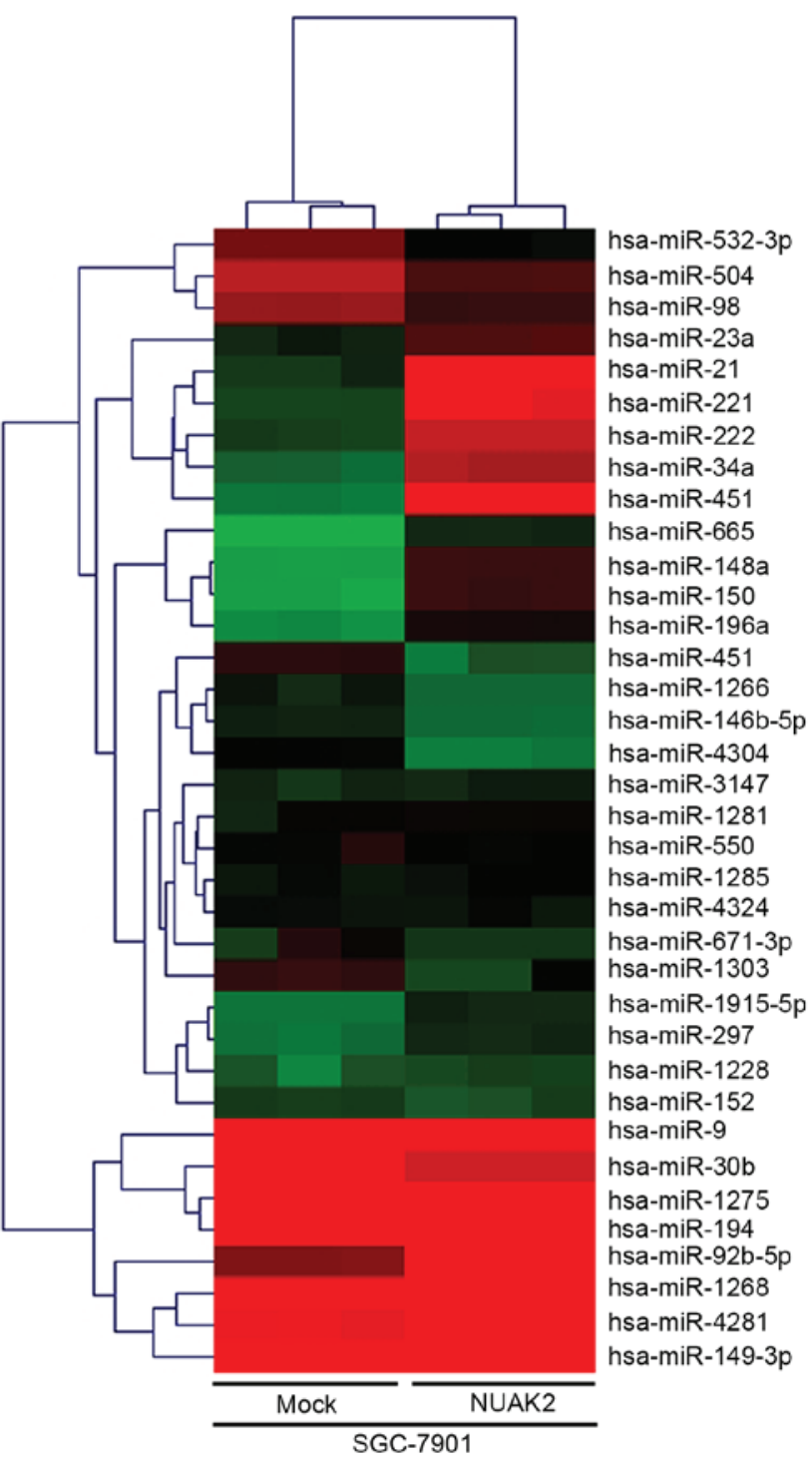

Figure 4. NUAK2 regulates proliferation and cancer stem cell-associated microRNAs. Microarray analysis of SGC-7901 cells transfected with NUAK2-expressing plasmids or empty vector (mock). Genes with similar expression profiles were grouped together using hierarchical clustering and the resulting genes tree is shown. Color bar indicates magnitude of gene regulation, with green and red indicating repression and stimulation, respectively.

Figure 3. NUAK2 regulates proliferation and cancer stem cell-associated markers in SGC-7901 cells. Expression of c-myc, p21, p53, proliferating cell nuclear antigen (PCNA), cyclin-dependent kinase 2 (CDK2), aldehyde dehydrogenase 1 (ALDH1), CD44 and CD133 in SGC-7901 cells transfected with NUAK2-expressing plasmids or empty vector (mock), as determined by western blotting.

important roles in gastric cancer through the regulation of miRNAs $(20,21)$. Having demonstrated that NUAK2, as an oncogene, promoted proliferation of SGC-7901 cells, it was hypothesized that NUAK2 was able to regulate proliferation-associated miRNA expression. In an attempt

to identify that NUAK2 contributed to aberrant regulation of miRNA expression in SGC-7901 cells, miRNA profiling was performed in SGC-7901 cells transfected with NUAK2expressing plasmids or empty vector. RNAs isolated from the cells were hybridized to a custom miRNA microarray platform. Following three hybridization cycles, quantification, and normalization, the expression of 36 miRNAs was altered in the cells transfected with NUAK2-expressing plasmids. Notably, miRNA21, which confers cisplatin resistance in gastric cancer cells by regulating phosphatase and tensin homolog (22), was significantly upregulated following NUAK2 overexpression (Fig. 4). 


\section{Discussion}

Previous studies have demonstrated that several oncogenes and tumor suppressor genes are dysregulated in gastric cancer tissues or cell lines, and they have been shown to be associated with gastric cancer progression and disease outcome (23-25). The present study demonstrated that NUAK 2 overexpression may promote cell proliferation and cell-cycle progression in SGC-7901 cells. In addition, NUAK2 overexpression was observed to upregulate cancer stem cell markers, both protein and miRNAs, in SGC-7901 cells. Therefore, NUAK2 may be considered as an oncogene in the development of gastric cancer.

NUAK2 is an emerging oncogene that regulates cell cycle progression and cell migration in melanoma cells $(6,7)$. A previous study also indicated that NUAK2 and the PI3K signaling pathway coordinately control CDK2 by abrogating the growth of cutaneous melanomas (9). The results of the present study suggested that NUAK 2 may also act as an oncogene in gastric cancer. However, its underlying mechanisms require further investigations.

The cell cycle machinery has a pivotal role in regulating cell proliferation and tumor growth of cancer cells. A previous study demonstrated that NUAK2 regulates CDKs in melanoma cells (9). Concordant with these results, the present study demonstrated that NUAK2 overexpression upregulates CDK2 and PCNA expression levels in gastric cancer cells. Previous studies have suggested that tumors develop because of a rare subpopulation of cells, known as cancer stem cells (CSCs), within a tumor $(26,27)$. CSCs have been identified in numerous solid tumors and they have been shown to be promising for the development of anticancer drugs that are able to target all CSC subsets within a tumor to prevent recurrence $(28,29)$. The results of the current study also demonstrated that NUAK2 was able to regulate CSC-associated markers, proteins and miRNAs, indicating it may have an essential role in tumor development.

In conclusion, the present study provides a novel role for NUAK2 in gastric cancer cell proliferation and regulation. These results suggest that NUAK2 may be a promising therapeutic target for gastric cancer therapy.

\section{Acknowledgements}

This study was supported by the Technology Development Foundation of Pudong District (grant no. PKJ2013-Y67 and PKJ2016-Y54), the Experimental Animal Special Purpose Foundation of Science and the Technology Commission of Shanghai Municipality (grant no. 13140902901).

\section{References}

1. Siegel R, Naishadham D and Jemal A: Cancer statistics, 2013. CA Cancer J Clin 63: 11-30, 2013.

2. Resende C, Thiel A, Machado JC and Ristimäki A: Gastric cancer: Basic aspects. Helicobacter 16 (Suppl 1): S38-S44, 2011.

3. Gomceli I, Demiriz B and Tez M: Gastric carcinogenesis. World J Gastroenterol 18: 5164-5170, 2012.

4. Ooi CH, Ivanova T, Wu J, Lee M, Tan IB, Tao J, Ward L, Koo JH, Gopalakrishnan V, Zhu Y, et al: Oncogenic pathway combinations predict clinical prognosis in gastric cancer. PLoS Genet 5: e1000676, 2009.

5. Van Cutsem E, Sagaert X, Topal B, Haustermans K and Prenen H: Gastric cancer. Lancet S0140-6736: 30354-3, 2016.
6. Yamamoto H, Takashima S, Shintani Y, Yamazaki S, Seguchi O, Nakano A, Higo S, Kato H, Liao Y, Asano Y, et al: Identification of a novel substrate for TNFalpha-induced kinase NUAK2. Biochem Biophys Res Commun 365: 541-547, 2008.

7. Namiki T, Coelho SG and Hearing VJ: NUAK2: An emerging acral melanoma oncogene. Oncotarget 2: 695-704, 2011.

8. Namiki T, Tanemura A, Valencia JC, Coelho SG, Passeron T, Kawaguchi M, Vieira WD, Ishikawa M, Nishijima W, Izumo T, et al: AMP kinase-related kinase NUAK2 affects tumor growth, migration and clinical outcome of human melanoma. Proc Natl Acad Sci USA 108: 6597-6602, 2011.

9. Namiki T, Yaguchi T, Nakamura K, Valencia JC, Coelho SG, Yin L, Kawaguchi M, Vieira WD, Kaneko Y, Tanemura A, et al: NUAK2 amplification coupled with PTEN deficiency promotes melanoma development via CDK activation. Cancer Res 75: 2708-2715, 2015.

10. Chen F, Tian Y, Pang EJ, Wang Y and Li L: MALAT2-activated long noncoding RNA indicates a biomarker of poor prognosis in gastric cancer. Cancer Gene Ther, 2015

11. Lang J, Lan X, Liu Y, Jin X, Wu T, Sun X, Wen Q and An R: Targeting cancer stem cells with an 131I-labeled anti-AC133 monoclonal antibody in human colorectal cancer xenografts. Nucl Med Biol 42: 505-512, 2015.

12. Tang L, Chen F, Pang EJ, Zhang ZQ, Jin BW and Dong WF: MicroRNA-182 inhibits proliferation through targeting oncogenic ANUBL1 in gastric cancer. Oncol Rep 33: 1707-1716, 2015.

13. Dalby B, Cates S, Harris A, Ohki EC, Tilkins ML, Price PJ and Ciccarone VC: Advanced transfection with Lipofectamine 2000 reagent: Primary neurons, siRNA, and high-throughput applications. Methods 33: 95-103, 2004.

14. Liao XH, Lu DL, Wang N, Liu LY, Wang Y, Li YQ, Yan TB, Sun $X G, H u P$ and Zhang TC: Estrogen receptor alpha mediates proliferation of breast cancer MCF-7 cells via a p21/PCNA/E2F1-dependent pathway. FEBS J 281: 927-942, 2014.

15. Dee S and Getts RC: MicroRNA expression analysis using the Affymetrix Platform. Methods Mol Biol 822: 117-129, 2012.

16. Wakamatsu Y, Sakamoto N, Oo HZ, Naito Y, Uraoka N, Anami K, Sentani K, Oue N and Yasui W: Expression of cancer stem cell markers ALDH1, CD44 and CD133 in primary tumor and lymph node metastasis of gastric cancer. Pathol Int 62: 112-119, 2012.

17. Yang L, Ping YF, Yu X, Qian F, Guo ZJ, Qian C, Cui YH and Bian XW: Gastric cancer stem-like cells possess higher capability of invasion and metastasis in association with a mesenchymal transition phenotype. Cancer Lett 310: 46-52, 2011.

18. Zhao P, Li Y and Lu Y: Aberrant expression of CD133 protein correlates with $\mathrm{Ki}-67$ expression and is a prognostic marker in gastric adenocarcinoma. BMC Cancer 10: 218, 2010

19. Fukamachi H, Shimada S, Ito K, Ito Y and Yuasa Y: CD133 is a marker of gland-forming cells in gastric tumors and Sox17 is involved in its regulation. Cancer Sci 102: 1313-1321, 2011.

20. Gregory RI and Shiekhattar R: MicroRNA biogenesis and cancer. Cancer Res 65: 3509-3512, 2005.

21. Gao C, Zhang Z, Liu W, Xiao S, Gu W and Lu H: Reduced microRNA-218 expression is associated with high nuclear factor kappaB activation in gastric cancer. Cancer 116: 41-49, 2010.

22. Yang SM, Huang C, Li XF, Yu MZ, He Y and Li J: MiR-21 confers cisplatin resistance in gastric cancer cells by regulating PTEN. Toxicology 306: 162-168, 2013.

23. Cancer Genome Atlas Research Network: Comprehensive molecular characterization of gastric adenocarcinoma. Nature 513: 202-209, 2014

24. Ott K, Vogelsang H, Mueller J, Becker K, Müller M, Fink U, Siewert JR, Höfler H and Keller G: Chromosomal instability rather than p53 mutation is associated with response to neoadjuvant cisplatin-based chemotherapy in gastric carcinoma. Clin Cancer Res 9: 2307-2315, 2003.

25. Sunakawa Y and Lenz HJ: Molecular classification of gastric adenocarcinoma: Translating new insights from the cancer genome atlas research network. Curr Treat Options Oncol 16: 17, 2015.

26. Ajani JA, Song S, Hochster HS and Steinberg IB: Cancer stem cells: The promise and the potential. Semin Oncol 42 Suppl 1: S3-17, 2015.

27. Zhao Y, Feng F and Zhou YN: Stem cells in gastric cancer. World J Gastroenterol 21: 112-123, 2015.

28. Visvader JE and Lindeman GJ: Canscer stem cells: Current status and evolving complexities. Cell Stem Cell 10: 717-728, 2012.

29. Singh SR: Gastric cancer stem cells: A novel therapeutic target. Cancer Lett 338: 110-119, 2013. 